\title{
Correspondence
}

\section{COVID-19 in Hematologic Malignancies: Big Challenges}

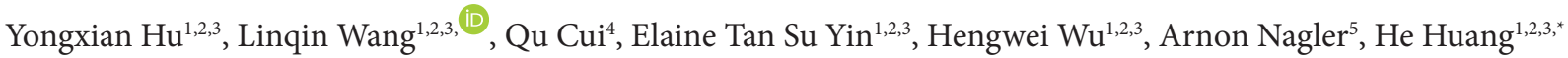 \\ ${ }^{1}$ Bone Marrow Transplantation Center, The First Affiliated Hospital, School of Medicine, Zhejiang University, Hangzhou, China \\ ${ }^{2} Z$ hejiang Province Engineering Laboratory for Stem Cell and Immunity Therapy, Hangzhou, China \\ ${ }^{3}$ Institute of Hematology, Zhejiang University, Hangzhou, China \\ ${ }^{4}$ Department of Hematology, Beijing Tiantan Hospital, Capital Medical University, Beijing, China \\ ${ }^{5}$ Hematology and Bone Marrow Transplantation Division, Chaim Sheba Medical Center, Tel-Hashomer, Israel
}

\section{ARTICLE INFO \\ Article History \\ Received 29 May 2020 \\ Accepted 16 September 2020 \\ Keywords \\ COVID-19 \\ hematological malignancies \\ clinical manifestation \\ treatment strategies}

(C) 2020 International Academy for Clinical Hematology. Publishing services by Atlantis Press International B.V. This is an open access article distributed under the CC BY-NC 4.0 license (http://creativecommons.org/licenses/by-nc/4.0/).
The Coronavirus Disease 2019 (COVID-19) became by now an overwhelming pandemic all over the world. Up to July 23, 2020, a total of more than 14,971,036 cumulative confirmed cases and 618,017 deaths have been reported globally, spanning more than 200 countries and regions. In response to the unprecedented outbreak of COVID-19, a number of rapid epidemiologic studies have revealed that fever and respiratory symptoms, such as nonproductive cough and dyspnea, represent the most common manifestations of COVID-19 [1]. Moreover, the Severe Acute Respiratory Syndrome Coronavirus 2 (SARS-CoV-2), the pathogen of COVID-19, was identified in stool, sputum, tears, urine and blood, which was firmly indicative of droplet and contact transmission, and implied the high infectivity of COVID-19 [1]. Notably, through binding to angiotensin-converting enzyme 2, SARS-CoV-2 is capable of inducing multiple organ dysfunction syndromes besides respiratory failure [2]. The severity and clinical outcome of COVID-19 varies among individuals. Zhou et al. [3] reported that increased odds of in-hospital death were associated with older age, higher Sequential Organ Failure Assessment score, and elevated level of $\mathrm{D}$-dimer on admission. Besides, advanced age has been reported as an important and independent predictor of mortality during the epidemic period of other coronaviruses, including SARS-CoV and Middle East Respiratory Syndrome (MERS)-CoV. Age-dependent defects in T-cell and B-cell biofunction and excessive production of type 2 cytokines, contribute to a deficiency in control of viral replication and prolonged proinflammatory responses.

"Corresponding author. Email: huanghe@zju.edu.cn

Peer review under responsibility of the International Academy for Clinical Hematology
The incidence of Hematologic Malignancies (HM) increases in older patients [4]. Fortunately, their survival has been remarkably ameliorated due to the introduction of chemotherapy, Hematopoietic Stem Cell Transplantation (HSCT), targeting therapy, and immunotherapy. Yet, these patients usually bear compromised immunity due to the disease per se and subsequent routine therapies, which expose them to high risks of severe SARS-CoV-2 infection. What is worse, during COVID-19, the early clinical manifestations in immunocompromised patients are less typical than in their immunocompetent counterparts. Furthermore, these HM patients are also a potential source of COVID-19 transmission. Thus, hematologists should promptly conduct early diagnosis, isolation and intervention when facing HM patients with COVID-19.

Most of these patients display the typical clinical manifestations, although some presented less common and more delayed symptoms than others. Based on the report from Saint-AntoineHospital, among 25 confirmed patients with HMs, fever (89\%), cough $(79 \%)$, and shortness of breath (79\%) are the most common symptoms [5]. However, Wang et al. [6] reported that a patient with multiple myeloma complained only of chest tightness and shortness of breath during the whole period of COVID-19 infection. Another case with mantle cell lymphoma and COVID-19 also showed no fever, as reported by Playe et al. [7]. In these situations, incompetent immune cells may release decreased levels of endogenous pyrogens, with the consequence that the body temperature probably does not elevate as markedly as in immune-competent individuals. More importantly, lymphoma-related fever is also deceptive, which may prevent the suspicion of COVID-19 as a concomitant disease. 
A second important aspect of COVID-19 in HM patients is the fact that their laboratory results are mostly consistent with those from individuals without comorbidities. In a comparative study of SARSCoV-2-infected HM patients and healthcare providers, no significant difference of leukocyte, neutrophil, alanine aminotransferase, aspartate aminotransferase was observed between them. However, in HM patients, the platelet count, lymphocyte count, the hemoglobin concentration, and the level of C-reactive protein, were significantly lower than in healthcare providers [8]. Typical laboratory results in COVID-19 include lymphocytopenia, followed by thrombocytopenia and/or leukopenia. Furthermore, elevated levels of C-reactive protein, alanine aminotransferase, aspartate aminotransferase, creatine kinase, and D-dimer are fairly common in severe COVID-19 cases $[3,9]$. However, in patients with a high tumor burden, the reduction of leukocyte and lymphocyte would be offset by expanded malignant cells. As reported, a Chinese male patient with Chronic Lymphoblastic Leukemia (CLL) affected by COVID-19, presented with a leukocyte count of $91.85 \times 10^{9} / \mathrm{L}$ and $96 \%$ lymphocytes upon admission [10]. Moreover, persistent agranulocytosis after chemotherapy could interfere with the medical judgment of underlying SARS-CoV-2 infection. In addition to that, Hemophagocytic Lymphohistiocytosis (HLH), characterized by a reduced level of trilineage cell lines, can be induced by malignant or viral infectious stimulus [11]. Among lymphoma patients, initial suspicion of lymphoma-based HLH would also mask the coexistence of COVID-19. Intriguingly, recent studies revealed the involvement of SARS-CoV-2 in endothelial cells accompanied with endotheliitis in COVID-19, which may induce microvascular thrombosis and procoagulant state $[12,13]$. Additionally, Wang et al. [14] reported the occurrence of disseminated intravascular coagulation in two severe COVID-19 patients which manifested elevated D-dimer, decreased platelet count and prolonged prothrombin time. It is worth cautioning that, during the regular follow-up of Acute Promyelocyte Leukemia (APL), the potential causes of coagulation dysfunction should be carefully differentiated between progression of APL and COVID-19 infection. During the outbreak of MERS [15], a female with recurring B-cell lymphoma received an autologous transplant. After her exposure to the infected patient, the MERS-CoV reverse transcription-polymerase chain reaction (RT-PCR) of sputa remained negative for 20 days, longer than the median incubation period, which may have been associated with her immunocompromised status induced by multiple courses of chemotherapy and autologous transplant. Thus, the rationale and timing of sputum RT-PCR for diagnosis of coronavirus infection deserve more careful considerations.

Thirdly, patients with COVID-19 showed ground-glass opacity and bilateral patchy shadowing as the most common radiologic patterns on chest Computerized Tomography (CT) [9]. However, due to the defective function of the T-cell or the monocyte/macrophage system, the imagining results in HM individuals may deviate from the typical presentations. Slighter manifestations may be seen in cases with coronavirus as the sole infection, but their radiographic patterns may be confounded by co-infectious agents, such as bacterial and fungal pathogens [16]. In Wuhan, a child was reported by Chen et al. [12] who was infected with SARS-CoV-2 after chemotherapy for acute lymphoblastic leukemia. During the anti-infective and supportive therapies, his chest radiologic findings converted from initial signs of lung inflammation and lung consolidation to floc-density shadow and ground-glass opacities, suggesting the occurrence of multiple or successive infections.
Fourthly, the incubation period for SARS-CoV-2 and the disease course of COVID-19 can be prolonged in a background of HM. Jin et al; [10] reported on a patient with CLL in whom COVID-19 developed with an incubation period of 25 days, possibly due to his immune-suppressed status. It is conceivable that a similar scenario takes place in patients with Multiple Myeloma (MM), the majority of whom have with immune paresis and heavy immunosuppression. In lymphoblastic leukemia, the immature or abnormal lymphocytes render the clearance of coronavirus less potent. Moreover, in other types of HM, the production of normal leukocytes can be perturbed by malignant cells in the bone marrow microenvironment. Consequently, SARS-CoV-2 probably maintains more persistent infectivity in these patients.

Treatment strategies to tackle the coexistence of COVID-19 and HM should be taken into consideration with adjusted chemotherapy doses, transplant protocols, Chimeric Antigen Receptor T cell (CAR-T) therapy regimens and enhanced supportive measures.

During the pandemic, chemotherapeutic regimens for HM should be reconsidered according to the stage and the type of cancers in order to exert minimal influence on the immune system. Acute myeloid leukemia patients would benefit from standard induction chemotherapy, and midostaurin should be added for those with Fms-like tyrosine kinase 3 (FLT3)-internal tandem duplication (ITD) mutation. For consolidation, the dose of cytarabine could be reduced without significant impact on the 5-year survival [17]. For acute lymphoblastic leukemia, the recommended dose of glucocorticoids should be used as a part of regimens [17]. For multiple myeloma, an induction regimen with up to six cycles of induction could allow postponement of treatment with high-dose melphalan and autologous HSCT, which are prone to induce a long period of immune dysfunction [18].

In the case of allogeneic HSCT, administration of granulocytecolony stimulating factor can, theoretically, aggravate an infected donor's lung injury by COVID-19 via cytokines and leukostasis. Thus, detailed virus screenings are recommended in each donor before stem cell mobilization. Moreover, non-urgent allogeneic transplants (for genetic diseases and non-HM) as well as autologous transplants (for $\mathrm{MM}$ and lymphoma) should better be postponed. Graft versus Host Disease (GVHD) will cause immunosuppression and increase the risk of COVID-19. Therefore, allo-HSCT with a lower incidence of GVHD and better immune reconstitution such as match-related and nonmyeloablative transplants should be preferred.

As for conducting CAR-T therapy, CAR-T cells are recommended to be cryopreserved in the hospital in advance to avoid unexpected delays during transport. Timely IV immunoglobulin replacement is also encouraged after CAR-T infusion. Moreover, given the severity of COVID-19 in the immunocompromised population, more intensive supportive interventions are advised to stabilize blood oxygen saturation, and balance intestinal microecology which are more critical than antiviral therapy in this potentially high-risk group [19].

In a nutshell, we discussed the potentially atypical and unconventional symptoms, laboratory results and radiologic patterns of COVID-19 in the background of HM, and also recommended a set of pertinent therapeutic strategies. Due to the aberrant immune background and quantitively abnormal leukocytes in HM, clinical and biochemical data of 
COVID-19 can be masked to a large extent, which increases the risk of missed diagnosis. Therefore, diagnostic and therapeutic strategies should be correspondingly and urgently optimized in these patients, in the form of reduced-dose regimens and more intensive interventions. Amid an ongoing pandemic of COVID-19, more caution and precautions should be included in the diagnosis and clinical management of these immunocompromised populations.

\section{CONFLICTS OF INTEREST}

The authors declare they have no conflicts of interest.

\section{AUTHORS' CONTRIBUTION}

$\mathrm{HH}$ took the lead in writing the manuscript. $\mathrm{YH}$ and $\mathrm{LW}$ wrote and edited the manuscript. QC, ETSY, HW and AN edited the manuscript. All authors read and approved the final manuscript and, therefore, had full access to all the data in the study and take responsibility for the integrity and security of the data.

\section{REFERENCES}

[1] Gu J, Han B, Wang J. COVID-19: gastrointestinal manifestations and potential fecal-oral transmission. Gastroenterology 2020;158;1518-19.

[2] Wang H, Zhang L. Risk of COVID-19 for patients with cancer. Lancet Oncol 2020;21;e181.

[3] Zhou F, Yu T, Du R, Fan G, Liu Y, Liu Z, et al. Clinical course and risk factors for mortality of adult inpatients with COVID-19 in Wuhan, China: a retrospective cohort study. Lancet 2020;395;1054-62.

[4] Kasamon YL, Bolaños-Meade J, Prince GT, Tsai HL, McCurdy SR, Kanakry JA, et al. Outcomes of nonmyeloablative HLAhaploidentical blood or marrow transplantation with high-dose post-transplantation cyclophosphamide in older adults. J Clin Oncol 2015;33;3152-61.

[5] Malard F, Genthon A, Brissot E, van de Wyngaert Z, Marjanovic Z, Ikhlef S, et al. COVID-19 outcomes in patients with hematologic disease. Bone Marrow Transplant 2020;1-5.

[6] Zhang X, Song K, Tong F, Fei M, Guo H, Lu Z, et al. First case of COVID-19 in a patient with multiple myeloma successfully treated with tocilizumab. Blood Adv 2020;4;1307-10.
[7] Playe M, Siavellis J, Braun T, Soussan M. FDG PET/CT in a patient with mantle cell lymphoma and COVID-19: typical findings. Clin Nucl Med 2020;45;e305-e6.

[8] He W, Chen L, Chen L, Yuan G, Fang Y, Chen W, et al. COVID-19 in persons with haematological cancers. Leukemia 2020;34;1637-45.

[9] Guan WJ, Ni ZY, Hu Y, Liang WH, Ou CQ, He JX, et al. Clinical characteristics of coronavirus disease 2019 in China. N Engl J Med 2020;382;1708-20.

[10] Jin XH, Zheng KI, Pan KH, Xie YP, Zheng MH. COVID-19 in a patient with chronic lymphocytic leukaemia. Lancet Haematol 2020;7;e351-e2.

[11] Al-Samkari H, Berliner N. Hemophagocytic lymphohistiocytosis. Annu Rev Pathol 2018;13;27-49.

[12] Varga Z, Flammer AJ, Steiger P, Haberecker M, Andermatt R, Zinkernagel AS, et al. Endothelial cell infection and endotheliitis in COVID-19. Lancet 2020;395;1417-18.

[13] Ciceri F, Beretta L, Scandroglio AM, Colombo S, Landoni G, Ruggeri A, et al. Microvascular COVID-19 lung vessels obstructive thromboinflammatory syndrome (MicroCLOTS): an atypical acute respiratory distress syndrome working hypothesis. Crit Care Resusc 2020;22;95-7.

[14] Wang YD, Zhang SP, Wei QZ, Zhao MM, Mei H, Zhang ZL, et al. Two cases report and literature review of novel coronavirus pneumonia (COVID-19) with diffuse intravascular coagulation. Chin J Haematol 2020;41;245-7.

[15] Kim SH, Ko JH, Park GE, Cho SY, Ha YE, Kang JM, et al. Atypical presentations of MERS-CoV infection in immunocompromised hosts. J Infect Chemother 2017;23;769-73.

[16] Vakil E, Evans SE. Viral pneumonia in patients with hematologic malignancy or hematopoietic stem cell transplantation. Clin Chest Med 2017;38;97-111.

[17] Brissot E, Labopin M, Baron F, Bazarbachi A, Bug G, Ciceri F, et al. Management of patients with acute leukemia during the COVID-19 outbreak: practical guidelines from the acute leukemia working party of the European Society for Blood and Marrow Transplantation. Bone Marrow Transplant 2020;1-4.

[18] Malard F, Mohty M. Management of patients with multiple myeloma during the COVID-19 pandemic. Lancet Haematol 2020;7;e435-e7.

[19] Liang T, Cai H, Chen Y, Chen Z, Fang Q, Han W, et al. Handbook of COVID-19 prevention and treatment. 2020. Available from: https://gmcc.alibabadoctor.com/prevention-manual/detail?content_id=0. 